\title{
ASSESSING THE PREVALENCE OF EYE DISEASE IN THE COMMUNITY
}

\author{
RICHARD WORMALD \\ London
}

The prevalence of any disease, condition or functional impairment is the proportion of the specifically defined population afflicted by that defined clinical state at any one point in time. The true prevalence is almost never known because it is neither possible nor efficient to examine an entire population instantaneously. Instead, prevalence is estimated using sample survey methodology, the validity of this method depending upon the representativeness of the sample for the defined population of interest, the principle of random sampling (that everyone in that population has an equal probability of being sampled) and the precision of case definition.

What determines prevalence? Incidence, the speed or force of morbidity, is the number of new cases of the defined condition arising in a defined population per unit time, most strictly termed person time at risk. The number of cases leaving the same population is determined by those who are cured or recover, who die or migrate. The balance of input and output determines prevalence. Thus, prevalence is of limited value in describing acute conditions of short duration, but for many of the common causes of blindness, which are both chronic and often avoidable, it is a useful measure of the efficacy of our attempts to intervene. The WHO global estimate of 17 million blinded by cataract, ${ }^{1}$ a figure rapidly increasing because the incidence of persons blinded by cataract is far outstripping intervention, ${ }^{2}$ powerfully illustrates this concept. An estimate of incidence, for which a well-controlled follow-up study is required, is more informative for the purposes of both health care planning and aetiological research. But an estimate of prevalence can be more easily obtained from a once-off cross-sectional study.

Prevalence is the same as the probability or risk that any given individual has that condition at one time point. The concept of relative risk is used when comparing different populations or subgroups within

Correspondence to: Richard Wormald, Moorfields Eye Hospital, City Road, London EC1V 2PD, UK. a population. When associated with different levels of exposure or risk factors, relative risk can be used to investigate disease aetiology, although adequate numbers of cases are required. Rarer diseases are better investigated by case-control methodology. Surveys have often formed the baseline for followup or longitudinal studies where the same sample population is revisited after a period, enabling the identification of new cases and incidence to be estimated.

Prevalence by definition can only be studied on a population basis where the number of individuals in the defined community is the denominator of the proportion. We cannot rely on routine statistics of patients presenting to hospital as a measure of the population burden of disease because there are many biases which influence use of hospital services and a precise population denominator cannot be defined. In many developing countries, males have better access to hospital services than females. Hospitalbased statistics would therefore lead to the conclusion that disease was more common in men, but a community-based study might show the opposite, women having a greater burden of disease because they have poorer access to health care. The blind and partial sight survey in the United Kingdom (previously the register), ${ }^{3}$ which is hospital-based, gives a very misleading view of both the number of visually impaired and the main causes. By no means all those eligible are registered and there is a strong bias towards untreatable causes of visual failure among those who are. Thus the commonest cause of registration is ageing maculopathy, while a population-based survey of visual impairment in the community finds cataract to be the commonest cause. $^{4}$

Another important function of cross-sectional studies is the planning and evaluation of health care, which has become of particular relevance in the United Kingdom since the changes in health service administration. 
A complete consideration of all the aspects of survey design and execution is beyond the scope and capacity of this article, but it is worth mentioning a few important points on case definition and some aspects of sampling strategy which are critical to a successful survey outcome.

\section{CASE DEFINITION}

Clinical conditions generally exist within a continuous spectrum extending from a disease-free to a totally morbid state. But for the purposes of counting, we need to categorise people as diseased or not diseased using a cut-off within that spectrum which must be applied consistently throughout the survey.

Ophthalmology presents some special problems, not least the fact that each individual has two eyes (which are not, statistically speaking, independent of one another). Do we count visual impairment by eye or individual? Another difficulty is the non-linear relationship between physical signs and visual function (e.g. lens opacity and ageing maculopathy). A study aimed at investigating the aetiology of a disease process requires a case definition independent of function, unlike one intended to inform planning and provision of services. Case definitions used in other studies should be considered so that direct comparisons can be made. Ultimately, case definition is determined by the research question and the intended use of the results, which must be anticipated at the planning stage.

Glaucoma is particularly problematic in case definition because of the difficulty of reliably diagnosing an early case. Most surveys have had to rely on a follow-up assessment to confirm or refute a suspected glaucoma diagnosis ${ }^{5}$ and the final case definition has depended on the agreement of two experienced ophthalmologists. Inevitably, glaucoma diagnosis in such circumstances errs towards the conservative.

The validity, reliability and acceptability of the tests which measure the condition of interest are critical in this regard. The cut-off criteria should be set such that the specificity of a test is high, if necessary at the expense of sensitivity. There is an important ethical issue here. The survey team exposes the sample to the possibility of having a previously undetected condition identified. In the clinical environment, a patient presents to the doctor with a complaint seeking diagnosis and treatment. To label erroneously an individual as diseased when no complaint is presented is to be avoided at all costs. It also is unethical to diagnose a condition in a survey without the wherewithal to provide treatment and follow-up.

The demands of survey instrumentation are high. Invasive and complex tests will have a disastrous affect on the response rate. And, because the tests will almost certainly need to be delivered in (usually) several different community settings, the instrumentation needs to be robust and technically reliable. This will often limit the sensitivity of any test. Any variation in measurement has to be kept to a minimum and each test used should be subjected to assessment of variation both between (inter-observer) and within (intra-observer) each observer/ operator. Inherent variation from the apparatus itself must be controlled by regular calibration. Because there may be only one opportunity to test a participant, a learning curve by operator or participant is unacceptable. Thus threshold visual field testing is impossible in a survey setting. In the end, a trade-off between what is desirable and what is practical determines case definition strategy.

\section{SAMPLING CONSIDERATIONS}

The target population must be precisely defined by factors such as age, sex and ethnic group. Other considerations such as ecology and disease status (for example a survey of diabetics) may also apply. Then a sampling frame is needed (a list of everyone in the target population which can be randomly sampled). The availability of such a frame is highly variable. In developing countries it is usually necessary to perform a local census, ${ }^{6}$ while in the West a number of different approaches are possible. Electoral registers are useful when they are regularly and reliably updated (as in the Republic of Ireland) ${ }^{7}$ and a population sample of adults is required. Schools are useful for children, and general practice lists, especially now that many are computerised and hold information on age and sex, ${ }^{3}$ are also valuable. However, consideration has to be given to the representativeness of the general practice and, ideally, the practices themselves should be randomly sampled. Postcode sampling is an attractive option in the United Kingdom, residential codes being listed and including only a few residences each, but a lot of leg-work is required with door-to-door visiting to identify the sample. Cluster sampling (taking whole communities) has many practical advantages but thought must be given to the possibility of disease clustering in the communities themselves and allowance may need to be made for this in the precision of final estimates by using a 'design effect' which may necessarily widen the confidence limits around an estimate of prevalence.

Sampling in Western populations, particularly urban ones, is a highly complex issue. Help can be obtained from professionals who sample populations for other purposes (political polling or market research $)^{4}$ but such expertise is not cheap. Advice on a sampling strategy can be obtained and this at least is worth taking. 
Sample size is determined by the required precision of the estimate (a disease of low prevalence requires greater precision), an idea of the expected prevalence and the sampling strategy. Cluster and multistage strategies require larger samples. A small increase in precision or reduction of the sample size can be achieved with stratified sampling. What is actually affordable and achievable is probably the most important arbiter of sample size.

\section{RESPONSE/NON-RESPONSE}

The most important bias in any survey arises from the response of the sample. A 'healthy volunteer' effect is usually seen whereby responders tend to be individuals who are aware and concerned about their health. If a person has already been diagnosed as irremediably visually impaired, it is unlikely that they will willingly subject themselves to another eye examination only to be given the same depressing conclusion. If someone is already under the care of an ophthalmologist, there is a tendency to refuse an offer of an alternative assessment.

Ideally, a response rate of over $80 \%$ is required, although in practice, especially in industrialised populations, this can be very hard to achieve. Chasing up non-responders is the hardest work of a survey and incentives may be required to reach an acceptable response rate. Visiting participants in their homes is nearly always essential, especially when elderly people are targeted. Being housebound with multiple comorbidity is an important risk factor for visual impairment. ${ }^{3}$

When all else fails, it is essential to have some information on the characteristics of the nonresponders. In the Inner City Eye Study we were able to consult general practice notes and discover that there was indeed a healthy volunteer effect. ${ }^{4}$

In summary, the principles of survey methodology are the same as those which apply throughout epidemiological research: case definition, measurement error and random sampling apply to all aspects of population-based research and apply to most hospital-based clinical research. This brief overview can only indicate the nature and complexity of these issues, which require study in further detail for those interested in undertaking epidemiological research.

\section{REFERENCES}

1. World Health Organization Programme Advisory Group. Report of the eighth meeting of the WHO Programme Advisory Group on the Prevention of Blindness. Geneva: World Health Organization, 1989. WHO publication 89.17.

2. Minassian DC, Mehra V. 3.8 Million blinded by cataract each year: projections from the first epidemiological study of incidence of cataract blindness in India. $\mathrm{Br} \mathbf{J}$ Ophthalmol 1990;74:341-3.

3. Government Statistical Service. Causes of blindness and partial sight among adults in 1976/77 and 1980/81. London: HMSO.

4. Wormald RPL, Wright LA, Courtney P, Beaumont B, Haines AP. Visual problems in the elderly population and implications for services. BMJ 1992;304:1226-9.

5. Tielsch JM, Katz J, Singh K, et al. A population-based evaluation of glaucoma screening: the Baltimore Eye Survey. Am J Epidemiol 1991;134:1102-10.

6. Faal H, Minassian A, Sowa A, Foster A. National survey of blindness and low vision in The Gambia: results. Br J Ophthalmol 1989;73:82-7.

7. Coffey M, Reidy A, Wormald R, Wu XX, Wright L, Courtney $P$. Prevalence of glaucoma in the west of Ireland. Br J Ophthalmol 1993;77:17-21. 